\title{
Avian Community Composition and Spatio- Temporal Patterns at Deva Vatala National Park, Azad Jammu and Kashmir, Pakistan
}

\author{
Muhammad Umar' ${ }^{1}$ Mubashar Hussain ${ }^{1 *}$, Muhammad Faheem Malik ${ }^{1}$, \\ Muhammad Naeem Awan² and David C. Lee ${ }^{3}$ \\ ${ }^{1}$ Department of Zoology, Faculty of Science, University of Gujrat, Gujrat, Pakistan \\ ${ }^{2}$ WWF Pakistan Regional Office Muzaffarabad, Pakistan \\ ${ }^{3}$ School of Applied Sciences, The University of South Wales, Pontypridd, UK
}

\begin{abstract}
A B S T R A C T
An assessment of the avifauna of Deva Vatala National Park (DVNP), Bhimber, Azad Jammu and Kashmir, Pakistan was conducted from June 2017 to May 2018. Data were collected along one km line transects at three sites within the National Park: Barmala, Deva and Vatala. In total, 52 species were recorded, which included the globally threatened sociable lapwing. The most abundant species were asian green bee-eater, red-vented bulbul, house sparrow and common myna, and no species was unique to a single site in the DVNP. Highest abundance, richness and diversity was recorded in Deva, with lowest community measures recorded in Barmala. Species richness and diversity peaked in September and was lowest in November. Applying a suite of community composition analyses, bird communities were significantly different across all the three sites (ANOSIM), with 10 species explaining $16.5 \%$ to $17.2 \%$ of these community dissimilarities (SIMPER). Of these key contributing species, the relative abundance of seven species was significantly different across sites. This study documents spatio-temporal patterns in the avifauna of DVNP, providing a basis for ongoing monitoring in the DVNP, and further studies focusing on bird-habitat associations and the current impacts of habitat degradation. The presence of sociable lapwing is an indication of habitat suitability and highlights the need for further surveys in the region to locate other possible wintering grounds for this critically endangered species.
\end{abstract}

\begin{tabular}{l} 
Article Information \\
Received 11 July 2019 \\
Revised 30 Auguat 2019 \\
Accepted 15 October 2019 \\
Available online 19 March 2021 \\
Authors' Contribution \\
\hline MU, MH and MFM conceived and \\
designed the study, collected the data \\
and drafted the manuscript. MNA and \\
DCL analysed the data and prepared \\
the final manuscript. \\
Key words \\
\hline Bird communities, PRIMER software, \\
Sociable lapwing
\end{tabular}

\section{INTRODUCTION}

$\mathrm{W}$ ith the current context of biodiversity losses, identifying changes in species and community composition is an important focus for supporting effective conservation management of protected area (Gamfeldt et al., 2008), which can play an integral role in biodiversity conservation (Ladin et al., 2016). This approach can assess existing biodiversity capital, current or anticipated drivers of impact, knowledge gaps and the directions required to improve management therein (Wathen et al., 2014). Continuous monitoring is a relatively cost-effective tool for protected area management (Gamfeldt et al., 2008). It can provide the basis for enhancing community engagement, education and outreach that supports longterm conservation goals (Berger et al., 2014).

Deva Vatala National Park (DVNP), Bhimber, Azad Jammu and Kashmir (AJ\&K) is located on the lower slopes of the Western Himalayas and, broadly, at a landscape ecotone between anthropogenically modified

\footnotetext{
* Corresponding author: dr.mubashar@uog.edu.pk 0030-9923/2021/0003-0921 \$9.00/0

Copyright 2021 Zoological Society of Pakistan
}

land and subtropical semi-evergreen forests (Grimmett et al., 2008). Historic conflict in the region has prevented any full appraisal of the National Park's conservation value. Consequently, an understanding of its biodiversity is lacking. Aut-ecological studies in the National Park have focused on Indian peafowl (Anwar et al., 2015) and Red jungle fowl (Subhani et al., 2010; Akrim et al., 2015; Anwar et al., 2016). However wider, community-based assessment of the avifauna in DVNP is lacking. This includes confirming species presence and any quantification of associated temporal or spatial distributions.

The aim of this study was to provide the first assessment of the avifauna in the National Park. We generate species' relative abundance and evaluate the spatio-temporal composition of the bird community within DVNP, providing a quantified basis for longterm monitoring. To this end, we utilise a suite of community composition analyses that may provide a template for similar studies in the region. We also present important records of the critically endangered Sociable lapwing, evidencing possible habitat suitability for this species. 


\section{MATERIALS AND METHODS}

Study area

The study was carried out at three sites (Barmala, Deva and Vatala) in Deva Vatala National Park (DVNP; $32^{\circ} 51-32^{\circ} 55 \mathrm{~N}, 74^{\circ} 16-74^{\circ} 24 \mathrm{E}$ ), AJ and K (Fig. 1), situated to the west of the line of control between Pakistan and India. Declared as a National Park in 2007, DVNP covers an area of 2,993 ha in the Western Himalayan foothills at an elevation of 267 to $536 \mathrm{~m}$ above sea level.

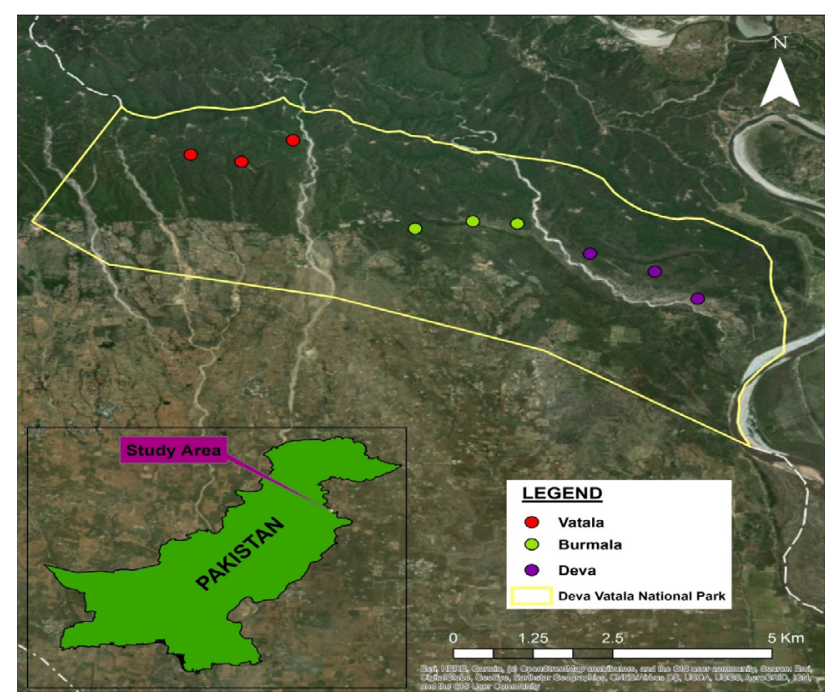

Fig. 1. Map of Deva Vatala National Park and the study site locations.

DVNP comprises sub-tropical semi-evergreen forests (Grimmett et al., 2008) and cultivated areas (e.g. for wheat; Anwar et al., 2015) over undulating terrain of the Deva and Vatala ranges (GOAJ\&K, 1985). Indicative plant species include Senegalia (Acacia) modesta, Dalbergia sissoo, Vachellia (Acacia) nilotica, Ficus benghalensis, Mangifera indica, Dodonaea viscosa, Carissa opaca, Ziziphus nummularia, Cynodon dactylon, Desmostachya bipinnata, and Saccharum spontaneum (Azam et al., 2007). With a dry sub-tropical climate, highest rainfall (974 mm) is in July and August, while annual temperatures range from $5^{\circ}$ to $46^{\circ} \mathrm{C}(\mathrm{GOAJ} \& \mathrm{~K}, 1985)$. In winter months, nomadic farmers move their livestock into DVNP for grazing, leaving again in the spring. Stone quarrying also poses a threat to biodiversity in DVNP.

Situated at an elevation of $350-411 \mathrm{~m}$ asl, the hilly forests of Barmala (32 52 '58.7' N, 74²0'18.97” E) have seasonal streams and, across vegetation layers, are dominated by Butea monosperma, D. sissoo, D. viscosa, Lannea coromandelica, S. spontaneum, V. nilotica, Salvia spp., Senna occidentalis, and Zanthoxylum armatum.
While comparatively undisturbed, livestock grazing, cutting wood for fuel, and grass collection and burning all impact upon the area. The forests of Deva (32 $54^{\prime} 8.6^{\prime \prime}$ $\mathrm{N}, 7^{\circ} 21^{\prime} 29.7^{\prime \prime} \mathrm{E} ; 306-381 \mathrm{~m}$ asl) include species characteristic of Barmala along with Aesculus indica, Ziziphus mauritiana, M. indica and Senegalia modesta (Subhani et al., 2010). Shrubs include Calotropis procera, D. viscosa, S. spontaneum, and Trichodesma indicum. This area of the DVNP has plains and seasonal streams. Human population density is lower than in Vatala but is impacted by daily movement of livestock to the forest areas, and vehicles transporting quarried stones. Situated closest to the line of control, the army's presence may also disturb this area of the park. Vatala $\left(32^{\circ} 52^{\prime} 38.7^{\prime \prime} \mathrm{N}, 7^{\circ} 17^{\prime} 44.7^{\prime \prime}\right.$ E; 350-396m asl) shares a similar plant community composition to the other sites, but is particularly dense with $D$. sissoo, $M$. indica, and D. viscosa. Human disturbance is the highest in this area, due to a higher population density, summer visitors, and the army, with most areas impacted by stone quarrying and livestock grazing. This has left only a few undistributed areas, mainly comprising open and cultivated areas.

\section{Bird survey methods}

The three study sites were each surveyed once a month from May 2017 to April 2018 using a fixedwidth line transect method. Three one $\mathrm{km}$ long transects were positioned randomly $>400 \mathrm{~m}$ apart in each of the three study areas. Surveys were conducted randomly in mornings (05:00-08:00 h) and afternoons (16:00-19:00 h). Each transect was surveyed by two observers walking at a speed of about $2 \mathrm{~km} / \mathrm{h}$ once in the morning or evening per month (36 transects per site across the study). All birds seen or heard along transect lines were recorded to a maximum perpendicular distance of $50 \mathrm{~m} ; 0.1 \mathrm{~km}^{2}$ surveyed per transect.

\section{Data analysis}

Species percentage relative abundance was converted into ordinal categories of abundance: only one individual recorded per month $=$ 'Rare'; 2-4 = 'Uncommon'; 5-9 = 'Frequent'; 10-19 = 'Common'; and $\geq 20=$ 'Abundant'. Numbers of independent encounters were converted to species' relative densities $\left(\mathrm{km}^{-2}\right)$, based on the total number of transects surveyed $(n=36)$, and assuming $100 \%$ detection probability of all birds within the $50 \mathrm{~m}$ fixedwidth (Buckland et al., 2001).

Site and overall species relative abundance were calculated for each species and tested using a one-away ANOVA, with site abundance compared using a Tukey post hoc test. Bird communities were quantified using a range of analyses in PRIMER v6.0 (Clarke and Gorley, 
2006). These techniques and their application in PRIMER are described fully in Clarke (1993), and Clarke and Warwick (2001). Each transect was factorised by site and month. The data were pre-treated with a square root transformation to down-weight the influence of the most abundant species (Clarke and Warwick, 2001). A similarity matrix was constructed using the Bray-Curtis coefficient. A similarity profile test (SIMPROF) was applied to a cluster analysis classification to identify clusters of samples with different community structures. These were ordinated using non-metric multidimensional scaling (nMDS). A two-way analysis of similarities (ANOSIM) was performed to investigate spatial and temporal differences between bird communities. A similarity of percentage analysis (SIMPER) was carried out to identify which species contributed the most to differences in communities across sites. The abundances of key contributing species were analysed using a Kruskal Wallis test, since the data did not fulfil the assumptions of parametric testing. A Bonferroni correction was used to adjust the significance values for groups of tests and avoid Type I errors. Overall and site-specific diversity indices were generated using the DIVERSE function in PRIMER (Clarke and Gorley, 2006). These were compared using a one-away ANOVA and Tukey post hoc testing.

\section{RESULTS}

\section{Overview}

In total, 6,487 birds of 52 species were recorded from 108 transect samples in DVNP (Table I). These included the globally threatened Sociable lapwing (CR; BirdLife International, 2018), which was recorded in all months except April, May, June and November, and with a peak count of five birds in August at Deva and Vatala. It was never recorded in Barmala. All 52 species were recorded in Deva, while $48(92.3 \%)$ were recorded in Vatala and $47(90.4 \%)$ in Barmala. Forty-four species $(84.6 \%)$ were common at all three sites, eight species (15.4\%) were observed at any two sites, while no species were unique to one site. Overall site diversity $\left(H_{\text {loge }}^{\prime}\right)$ was 3.307 , while location-specific species diversity was significantly higher in Deva $\left(H_{\text {loge }}^{\prime}=3.415 \pm 0.044\right)$ than in Vatala $\left(H^{\prime}{ }_{\text {loge }}=\right.$ $3.273 \pm 0.004)$ and Barmala $\left(H^{\prime}{ }_{\text {loge }}=3.232 \pm 0.032 ; F_{2,33}^{\text {loge }}=\right.$ 6.787, $P=0.003)$.

The most abundant species were Asian green beeeater, Red-vented bulbul, House sparrow and Common myna, accounting for $22.5 \%$ of overall community abundance. Thirty-seven species were residents, eight were winter visitors, six summer visitors, and one irregular visitor (Intermediate egret). Northern house martin and White wagtail were the most abundant summer and winter visitors, respectively. Rufous-tailed lark and Sociable lapwing were the least abundant wintering species, while Spotted forktail and Eurasian golden oriole were the least abundant summer breeders. Ten species were classified as Abundant, 10 as Common, 16 as Frequent, 12 as Uncommon (including Sociable lapwing) and four as Rare (Table I).

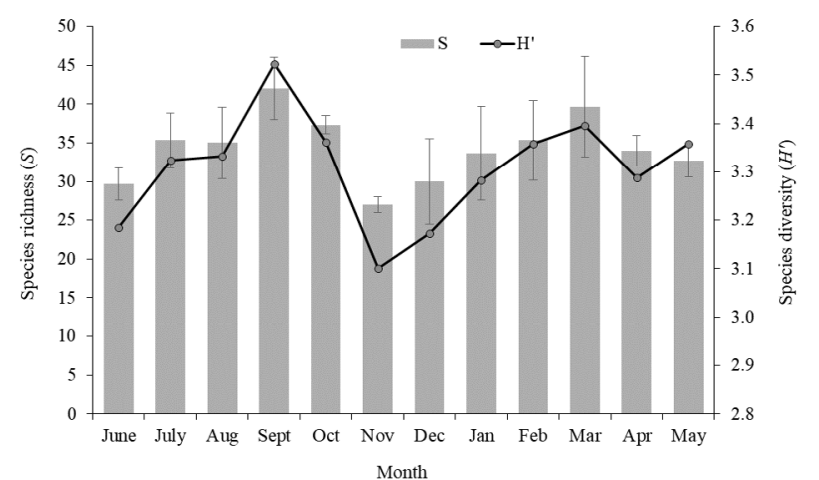

Fig. 2. Monthly species richness and diversity in Deva Vatala National Park.

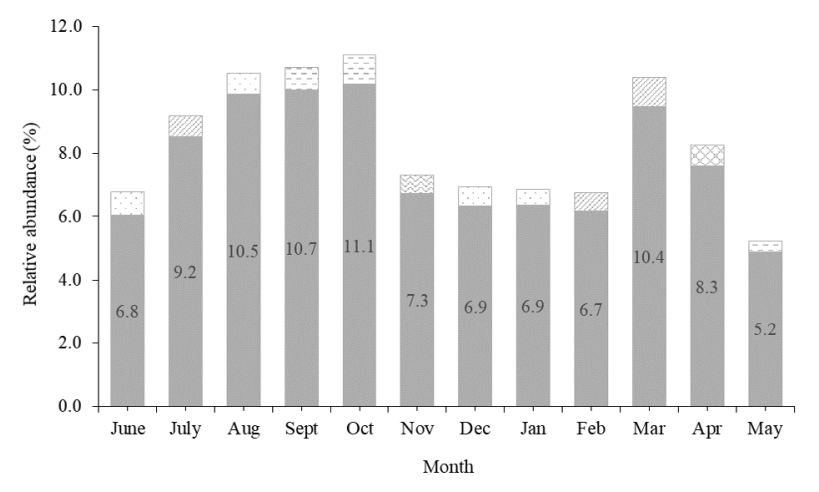

Fig. 3. Monthly relative abundance of total numbers of birds (\%) in Deva Vatala National Park. Total relative abundances are attached to the bars. The relative abundance of the most commonly recorded species per month are included.

\section{Spatio-temporal patterns in community composition}

Maximum mean species richness and diversity was recorded in September $\left(S_{\mathrm{r}}=42.0 \pm 4.00 ; H^{\prime}=3.52 \pm\right.$ $0.094)$, while the lowest species richness and diversity was recorded in November $\left(S_{\mathrm{r}}=27.0 \pm 1.00 ; H^{\prime}=3.10\right.$ \pm 0.045 ; Fig. 2). Relative abundance of birds recorded was the highest in October (11.1\%) and March (10.7\%), and lowest in May (6.5\%), although this did not change significantly across months (Fig. 3). The most frequently encountered species by months were: Common myna 
Table I. Species relative abundance and density $\left(\mathrm{km}^{-2}\right)$.

\begin{tabular}{|c|c|c|c|c|c|}
\hline Species & $\begin{array}{l}\text { Breeding } \\
\text { status }\end{array}$ & $\begin{array}{l}\text { Conservation } \\
\text { status }\end{array}$ & $\begin{array}{l}\text { Relative abun- } \\
\text { dance }(\%)\end{array}$ & $\begin{array}{l}\text { Abun- } \\
\text { dance }{ }^{1}\end{array}$ & $\begin{array}{l}\text { Relative den- } \\
\text { sity }( \pm \text { SE) }\end{array}$ \\
\hline Asian green bee-eater (Merops orientalis) & $\mathrm{R}$ & $\mathrm{LC}$ & 6.0 & A (386) & $3.2 \pm 0.20$ \\
\hline Red-vented bulbul (Pycnonotus cafer) & $\mathrm{R}$ & $\mathrm{LC}$ & 5.8 & A (377) & $3.1 \pm 0.20$ \\
\hline House sparrow (Passer domesticus) & $\mathrm{R}$ & $\mathrm{LC}$ & 5.4 & A (350) & $2.9 \pm 0.20$ \\
\hline Common myna (Acridotheres tristis) & $\mathrm{R}$ & $\mathrm{LC}$ & 5.3 & A (347) & $2.8 \pm 0.22$ \\
\hline Black drongo (Dicrurus macrocercus) & $\mathrm{R}$ & $\mathrm{LC}$ & 4.6 & $\mathrm{~A}(300)$ & $2.5 \pm 0.18$ \\
\hline Purple sunbird (Cinnyris asiaticus) & $\mathrm{R}$ & $\mathrm{LC}$ & 4.5 & A (293) & $2.4 \pm 0.22$ \\
\hline Common babbler (Argya caudata) & $\mathrm{R}$ & $\mathrm{LC}$ & 4.4 & A (284) & $2.3 \pm 0.20$ \\
\hline Himalayan bulbul (Pycnonotus leucogenys) & $\mathrm{R}$ & $\mathrm{LC}$ & 3.9 & $A(256)$ & $2.1 \pm 0.19$ \\
\hline Brown rockchat (Oenanthe fusca) & $\mathrm{R}$ & $\mathrm{LC}$ & 3.7 & A (242) & $2.0 \pm 0.17$ \\
\hline House crow (Corvus splendens) & $\mathrm{R}$ & $\mathrm{LC}$ & 3.7 & A (237) & $1.9 \pm 0.18$ \\
\hline Indian robin (Saxicoloides fulicatus) & $\mathrm{R}$ & $\mathrm{LC}$ & 3.2 & $\mathrm{C}(210)$ & $1.7 \pm 0.15$ \\
\hline Jungle babbler (Turdoides striata) & $\mathrm{R}$ & $\mathrm{LC}$ & 3.1 & C (199) & $1.6 \pm 0.15$ \\
\hline Oriental turtle-dove (Streptopelia orientalis) & $\mathrm{R}$ & $\mathrm{LC}$ & 2.8 & $\mathrm{C}(183)$ & $1.5 \pm 0.20$ \\
\hline Cattle egret (Bubulcus ibis) & $\mathrm{R}$ & $\mathrm{LC}$ & 2.8 & $\mathrm{C}(179)$ & $1.4 \pm 0.17$ \\
\hline Pied bushchat (Saxicola caprata) & $\mathrm{R}$ & $\mathrm{LC}$ & 2.6 & $\mathrm{C}(170)$ & $1.4 \pm 0.14$ \\
\hline Brahminy starling (Sturnia pagodarum) & $\mathrm{R}$ & $\mathrm{LC}$ & 2.5 & $\mathrm{C}(160)$ & $1.3 \pm 0.17$ \\
\hline Northern house martin (Delichon urbicum) & $\mathrm{S}$ & $\mathrm{LC}$ & 2.3 & $\mathrm{C}(147)$ & $1.2 \pm 0.18$ \\
\hline White wagtail (Motacilla alba) & $\mathrm{W}$ & $\mathrm{LC}$ & 2.1 & $\mathrm{C}(137)$ & $1.1 \pm 0.18$ \\
\hline Grey partridge (Perdix perdix) & $\mathrm{R}$ & $\mathrm{LC}$ & 2.0 & $\mathrm{C}(127)$ & $1.0 \pm 0.17$ \\
\hline Laughing dove (Spilopelia senegalensis) & $\mathrm{R}$ & $\mathrm{LC}$ & 1.9 & $\mathrm{C}(121)$ & $1.0 \pm 0.12$ \\
\hline Red-wattled lapwing (Vanellus indicus) & $\mathrm{R}$ & $\mathrm{LC}$ & 1.6 & $\mathrm{~F}(103)$ & $0.8 \pm 0.16$ \\
\hline Spotted flycatcher (Muscicapa striata) & $\mathrm{W}$ & $\mathrm{LC}$ & 1.5 & $\mathrm{~F}(100)$ & $0.8 \pm 0.15$ \\
\hline Indian peafowl (Pavo cristatus) & $\mathrm{R}$ & $\mathrm{LC}$ & 1.5 & F (99) & $0.8 \pm 0.12$ \\
\hline Graceful prinia (Prinia gracilis) & $\mathrm{R}$ & $\mathrm{LC}$ & 1.5 & $\mathrm{~F}(95)$ & $0.7 \pm 0.15$ \\
\hline Western spotted dove (Spilopelia suratensis) & $\mathrm{S}$ & $\mathrm{LC}$ & 1.4 & $\mathrm{~F}(91)$ & $0.7 \pm 0.12$ \\
\hline Common hoopoe (Upupa epops) & $\mathrm{R}$ & $\mathrm{LC}$ & 1.3 & $\mathrm{~F}(87)$ & $0.7 \pm 0.11$ \\
\hline White-eared bulbul (Pycnonotus leucotis) & $\mathrm{R}$ & $\mathrm{LC}$ & 1.3 & $\mathrm{~F}(86)$ & $0.7 \pm 0.14$ \\
\hline Oriental magpie-robin (Copsychus saularis) & $\mathrm{R}$ & $\mathrm{LC}$ & 1.2 & $\mathrm{~F}(77)$ & $0.6 \pm 0.13$ \\
\hline Crested lark (Galerida cristata) & $\mathrm{R}$ & $\mathrm{LC}$ & 1.1 & $\mathrm{~F}(74)$ & $0.6 \pm 0.13$ \\
\hline Indian roller (Coracias benghalensis) & $\mathrm{R}$ & $\mathrm{LC}$ & 1.1 & $\mathrm{~F}(71)$ & $0.5 \pm 0.10$ \\
\hline Variable wheatear (Oenanthe picata) & $\mathrm{R}$ & $\mathrm{LC}$ & 1.0 & $\mathrm{~F}(65)$ & $0.5 \pm 0.12$ \\
\hline Rufous treepie (Dendrocitta vagabunda) & $\mathrm{R}$ & $\mathrm{LC}$ & 1.0 & $\mathrm{~F}(63)$ & $0.5 \pm 0.11$ \\
\hline Tree pipit (Anthus trivialis) & $\mathrm{S}$ & $\mathrm{LC}$ & 0.9 & $\mathrm{~F}(61)$ & $0.5 \pm 0.11$ \\
\hline Red turtle-dove (Streptopelia tranquebarica) & $\mathrm{R}$ & $\mathrm{LC}$ & 0.9 & $\mathrm{~F}(61)$ & $0.5 \pm 0.14$ \\
\hline Red junglefowl (Gallus gallus) & $\mathrm{R}$ & $\mathrm{LC}$ & 0.9 & $\mathrm{~F}(60)$ & $0.5 \pm 0.10$ \\
\hline Jacobin cuckoo (Clamator jacobinus) & $\mathrm{R}$ & $\mathrm{LC}$ & 0.9 & $\mathrm{~F}(60)$ & $0.5 \pm 0.12$ \\
\hline
\end{tabular}




\begin{tabular}{|c|c|c|c|c|c|}
\hline Species & $\begin{array}{l}\text { Breeding } \\
\text { status }\end{array}$ & $\begin{array}{l}\text { Conservation } \\
\text { status }\end{array}$ & $\begin{array}{l}\text { Relative abun- } \\
\text { dance }(\%)\end{array}$ & $\begin{array}{l}\text { Abun- } \\
\text { dance }{ }^{1}\end{array}$ & $\begin{array}{l}\text { Relative den- } \\
\text { sity }( \pm \text { SE) }\end{array}$ \\
\hline Black bulbul (Hypsipetes leucocephalus) & $\mathrm{W}$ & LC & 0.8 & $\mathrm{U}(54)$ & $0.4 \pm 0.11$ \\
\hline Long-tailed shrike (Lanius schach) & $\mathrm{W}$ & $\mathrm{LC}$ & 0.8 & $\mathrm{U}(49)$ & $0.4 \pm 0.11$ \\
\hline Intermediate egret (Ardea intermedia) & I & $\mathrm{LC}$ & 0.7 & $\mathrm{U}(48)$ & $0.4 \pm 0.13$ \\
\hline White-breasted kingfisher (Halcyon smyrnensis) & $\mathrm{R}$ & $\mathrm{LC}$ & 0.7 & $\mathrm{U}(46)$ & $0.3 \pm 0.10$ \\
\hline Large grey babbler (Argya malcolmi) & $\mathrm{S}$ & $\mathrm{LC}$ & 0.7 & $\mathrm{U}(45)$ & $0.3 \pm 0.12$ \\
\hline Western koel (Eudynamys scolopaceus) & $\mathrm{R}$ & $\mathrm{LC}$ & 0.7 & $\mathrm{U}(43)$ & $0.3 \pm 0.10$ \\
\hline Indian cuckoo (Cuculus micropterus) & $\mathrm{W}$ & $\mathrm{LC}$ & 0.6 & $\mathrm{U}(37)$ & $0.3 \pm 0.08$ \\
\hline Eurasian collared-dove (Streptopelia decaocto) & $\mathrm{R}$ & $\mathrm{LC}$ & 0.5 & $\mathrm{U}(35)$ & $0.2 \pm 0.10$ \\
\hline Greater hoopoe-lark (Alaemon alaudipes) & $\mathrm{W}$ & $\mathrm{LC}$ & 0.5 & $\mathrm{U}(32)$ & $0.2 \pm 0.10$ \\
\hline White-bellied redstart (Hodgsonius phaenicuroides) & $\mathrm{R}$ & $\mathrm{LC}$ & 0.5 & $\mathrm{U}(31)$ & $0.2 \pm 0.11$ \\
\hline Hume's wheatear (Oenanthe albonigra) & $\mathrm{R}$ & $\mathrm{LC}$ & 0.4 & $\mathrm{U}(28)$ & $0.2 \pm 0.10$ \\
\hline Sociable lapwing (Vanellus gregarius) & $\mathrm{W}$ & $\mathrm{CR}$ & 0.4 & $\mathrm{U}(25)$ & $0.2 \pm 0.09$ \\
\hline Rufous-tailed lark (Ammomanes phoenicura) & $\mathrm{W}$ & $\mathrm{LC}$ & 0.3 & $\mathrm{R}(22)$ & $0.1 \pm 0.11$ \\
\hline Black francolin (Francolinus francolinus) & $\mathrm{R}$ & $\mathrm{LC}$ & 0.3 & $\mathrm{R}(17)$ & $0.1 \pm 0.08$ \\
\hline Spotted forktail (Enicurus maculatus) & $\mathrm{S}$ & $\mathrm{LC}$ & 0.2 & $\mathrm{R}(12)$ & $0.1 \pm 0.08$ \\
\hline Eurasian golden oriole (Oriolus oriolus) & $\mathrm{S}$ & $\mathrm{LC}$ & 0.1 & $\mathrm{R}(5)$ & $0.0 \pm 0.05$ \\
\hline
\end{tabular}

Breeding status: I (irregular visitor), R (resident), S (summer visitor), W (winter visitor). Conservation status: CR (Critically Endangered), LC (Least Concern). ${ }^{1}$ Abundance (ordinal scale), with total number of individuals encountered in parentheses: A, Abundant; C, Common; F, Frequent; U, Uncommon; R, Rare.

Table II. Main species contributing to dissimilarities among site communities.

\begin{tabular}{|c|c|c|c|c|c|}
\hline Species & $\begin{array}{l}\text { Mean abundance } \\
( \pm \text { SE; Site } 1)\end{array}$ & $\begin{array}{l}\text { Mean abundance } \\
( \pm \text { SE; Site } 2)\end{array}$ & $\begin{array}{l}\text { Mean dissimilarity } \\
( \pm \text { SD })\end{array}$ & $\%$ Contribution & $\begin{array}{l}\text { Cumulative } \\
\%\end{array}$ \\
\hline & Barmala & Deva & & & \\
\hline Oriental turtle dove & $0.9 \pm 0.30$ & $5.3 \pm 0.60$ & $1.3 \pm 1.48$ & 3.7 & 3.7 \\
\hline Red-wattled lapwing & 0 & $1.8 \pm 0.17$ & $1.3 \pm 3.90$ & 3.4 & 7.1 \\
\hline House crow & \pm 0.28 & $2.7 \pm 0.15$ & $1.2 \pm 1.51$ & 3.2 & 10.3 \\
\hline Purple sunbird & $2.4 \pm 0.45$ & $2.1 \pm 0.41$ & $1.1 \pm 1.18$ & 3.1 & 13.4 \\
\hline \multirow[t]{2}{*}{ Cattle egret } & $1.0 \pm 0.31$ & $2.5 \pm 0.14$ & $1.1 \pm 1.37$ & 3.1 & 16.5 \\
\hline & Barmala & Vatala & & & \\
\hline House crow & $1.1 \pm 0.28$ & $3.1 \pm 0.14$ & $1.5 \pm 1.89$ & 4.1 & 4.1 \\
\hline Red-wattled lapwing & 0 & $2.0 \pm 0.32$ & $1.4 \pm 1.86$ & 3.8 & 7.9 \\
\hline White wagtail & $1.0 \pm 0.30$ & $1.9 \pm 0.44$ & $1.2 \pm 1.32$ & 3.2 & 11.1 \\
\hline Cattle egret & $1.0 \pm 0.31$ & $2.4 \pm 0.27$ & $1.1 \pm 1.41$ & 3.1 & 14.2 \\
\hline \multirow[t]{2}{*}{ Oriental turtle-dove } & $0.9 \pm 0.30$ & $2.1 \pm 0.28$ & $1.1 \pm 1.46$ & 3.0 & 17.2 \\
\hline & Deva & Vatala & & & \\
\hline Common myna & $2.0 \pm 0.24$ & $4.0 \pm 0.19$ & $1.3 \pm 1.76$ & 3.8 & 3.8 \\
\hline Northern house martin & $0.7 \pm 0.30$ & $2.2 \pm 0.35$ & $1.2 \pm 1.52$ & 3.6 & 7.4 \\
\hline Brahminy starling & $1.0 \pm 0.31$ & $2.7 \pm 0.16$ & $1.1 \pm 1.44$ & 3.3 & 10.7 \\
\hline Himalayan bulbul & $1.5 \pm 0.28$ & $3.2 \pm 0.19$ & $1.1 \pm 1.55$ & 3.3 & 14.0 \\
\hline White wagtail & $1.5 \pm 0.35$ & $1.9 \pm 0.44$ & $1.0 \pm 1.25$ & 3.0 & 17.0 \\
\hline
\end{tabular}

Only the top five contributing species are listed for each pairwise comparison. Analysis is based on pre-treated square-root transformed abundance (Clarke and Warwick, 2001). 
(June, August, December and January), Purple sunbird (July, February and March), Red-vented bulbul (September, October and May), Black drongo (November), and Asian green bee-eater (April; Fig. 3). Overall monthly abundance of birds was significantly different across sites $\left(F_{(2,33)}=3.741, P=0.034\right)$, and significantly higher in Vatala $(202.8 \pm 12.52 \mathrm{SE})$ than Barmala (154.0 $\pm 11.09 \mathrm{SE})$.

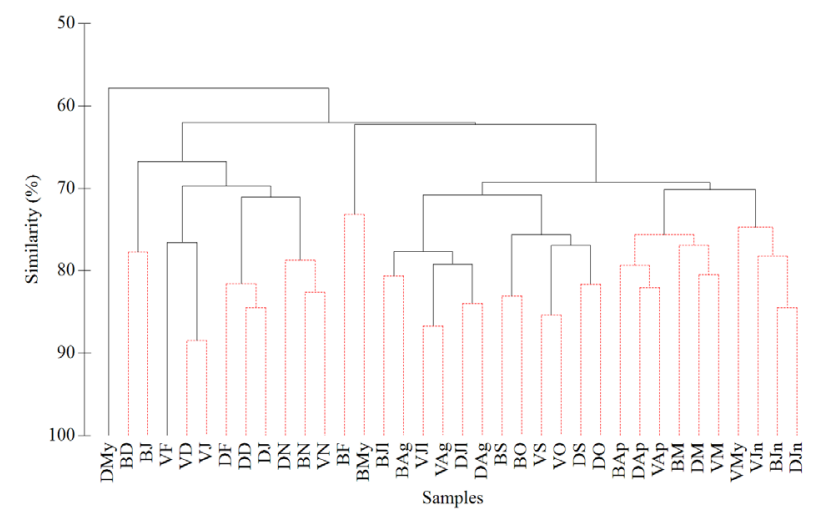

Fig. 4. Cluster analysis dendrogram of bird transect samples based on Bray-Curtis similarity values. Red dashed lines represent clusters with significant community structures using a SIMPROF analysis. Sample prefixes refer to the site, while suffixes refer to the month (e.g. 'DMy' = Species sample similarity from Deva in May, 2018).

The cluster analysis dendrogram (Fig. 4) and accompanying nMDS ordination plot (Fig. 5) characterised the temporal and spatial clustering of bird community compositions, which is broadly along seasonal groupings. All bird communities were at least $60 \%$ similar to one another, except for the May community in Deva. Bird communities were significantly different in composition across all sites (ANOSIM; $R=0.391, P=$ 0.001). All pairwise comparisons of bird communities between sites were significantly different (BarmalaDeva, $R=0.367, P=0.002$; Barmala-Vatala, $R=0.461$, $P=0.001)$; Deva-Vatala, $R=0.364, P=0.001$ ). Withinsite community composition similarities were between $67.6 \%$ (Barmala) and $72.3 \%$ (Vatala), while between-site dissimilarities ranged from $33.5 \%$ (Deva-Vatala) to $36.4 \%$ (Barmala-Deva). The top five species, represented by 10 species across all sites ( $21.2 \%$ of total species recorded), contributed $16.5 \%$ to $17.2 \%$ of community composition dissimilarities, although no single species contributed to dissimilarities between all three sites' bird communities (Table II). Of these key contributing species, the relative abundance of seven species was significantly different across sites (Table III; using a corrected $P$ value of 0.005 , where $k=10$ ). On average, key contributing species were significantly more abundant in Vatala (mean rank $=235.7)$ than in Deva (mean rank $=163.2)$ and Barmala (mean rank $\left.=142.7 ; H_{2}=53.70, P<0.001\right)$.

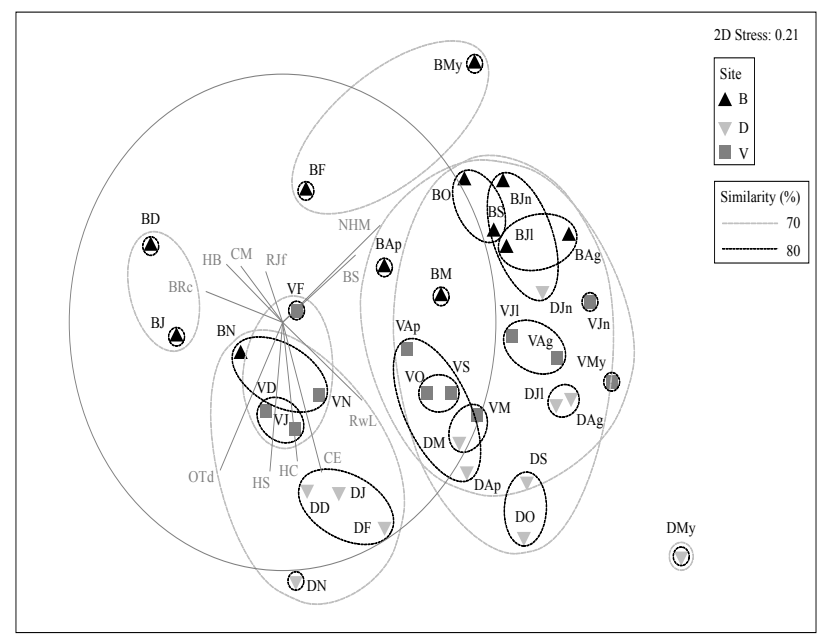

Fig. 5. nMDS ordination of samples with $70 \%$ and $80 \%$ similarity contours, and strength and direction vectors for key species displayed. Sample codes follow the nomenclature in Figure 4. Species codes: BRc, Brown rock chat; BS, Brahminy starling; $\mathrm{CE}$, Cattle egret; CM, Common myna; HB, Himalayan bulbul; HC, House crow; HS, House sparrow; NHM, Northern house martin; OTd, Oriental turtle dove; RJf, Red junglefowl; RwL, Redwattled lapwing.

Table III. Kruskal Wallis results of between-site differences in abundances of the top 10 key contributing species.

\begin{tabular}{|c|c|c|c|c|c|}
\hline Species & $\boldsymbol{H}$ & $P$ & Barmala & Deva & Vatala \\
\hline House crow & 23.67 & $<0.001$ & $\mathrm{a}$ & $\mathrm{b}$ & $\mathrm{b}$ \\
\hline Common myna & 21.25 & $<0.001$ & $\mathrm{a}$ & $\mathrm{a}$ & $\mathrm{b}$ \\
\hline Red-wattled lapwing & 21.10 & $<0.001$ & $\mathrm{a}$ & $\mathrm{b}$ & $\mathrm{b}$ \\
\hline Himalayan bulbul & 16.93 & $<0.001$ & a & $\mathrm{b}$ & $\mathrm{a}$ \\
\hline Brahminy starling & 16.27 & $<0.001$ & $\mathrm{a}$ & $\mathrm{a}$ & $\mathrm{b}$ \\
\hline Cattle egret & 12.56 & 0.002 & $\mathrm{a}$ & $\mathrm{b}$ & $\mathrm{b}$ \\
\hline Oriental turtle dove & 11.78 & 0.003 & $\mathrm{a}, \mathrm{c}$ & $\mathrm{b}$ & $\mathrm{b}, \mathrm{c}$ \\
\hline $\begin{array}{l}\text { Northern house } \\
\text { martin }\end{array}$ & 9.07 & 0.011 & & & \\
\hline White wagtail & 4.18 & 0.123 & & & \\
\hline Purple sunbird & 1.75 & 0.418 & & & \\
\hline
\end{tabular}

The shading indicates species' relative abundance rankings (light grey, site with lowest abundance; dark grey, site with highest abundance). Letters indicate similar or significantly different pairwise site abundances (with a Bonferroni correction). 


\section{DISCUSSION}

The most abundant species were, perhaps unsurprisingly, all resident birds, although only two of these could be considered forest species (Rasmussen and Anderton, 2005). Common myna is a common resident of open and agricultural areas (Snow and Perrins, 1998), as are Black drongo and Asian green bee-eater (MacKinnon and Phillipps, 2005), the latter avoiding wetter, higher elevation habitat (Snow and Perrins, 1998). Red-vented bulbul and Purple sunbird are associated with open forest (Rasmussen and Anderton, 2005), and the latter with scrub vegetation and forest edge (MacKinnon and Phillipps, 2005), being locally nomadic in response to nectar abundance (Grimmett et al., 2008).

Similarly, the seven significant species contributing to avian community dissimilarities across the DVNP were all resident that tend to be associated with more open, drier habitats: Red-wattled lapwing is associated with open areas adjacent to wetlands in cultivated and forest habitats (Wiersma and Kirwan, 2016); House crow is associated with anthropogenically-altered habitats throughout its range (Snow and Perrins, 1998); Cattle egret is specifically associated with livestock in dry grasslands (Grimmett et al., 2008); Himalayan bulbul is often found in drier valleys (MacKinnon and Phillipps, 2005); and although uncommon, Brahminy starling is also associated with human habitation (MacKinnon and Phillipps, 2005). All seven species were recorded at significantly lower abundance levels in Barmala than at one or both of the other two sites. Barmala is the least disturbed of the sites and, consequently, retains more forested vegetation, compared to the more open, impacted landscapes of Deva and Vatala. This spatial heterogeneity emphasises the importance of representatively surveying the landscape to get a truer reflection of the avian community, while changes in community composition provide quantifiable biodiversity metrics for supporting protected area conversation management (Gamfeldt et al., 2008).

The records of Sociable lapwing in DVNP represent suitability of habitat that is approximately $60 \mathrm{~km}$ east to the species' known wintering range (BirdLife International, 2018). While normally arriving at its wintering grounds from September (del Hoyo et al., 1996), five birds were recorded in August, and 1-4 birds were recorded monthly through to March. Based on a maximum monthly count of five birds, a site wintering density of 0.3 birds $\mathrm{km}^{2}$, and assuming equal distribution of birds throughout the Park, DVNP potentially holds a wintering population of 8-9 individuals $( \pm 3.0)$. While a comparatively small sub-population, this remains an important addition to conservation understanding of this declining, critically endangered species (BirdLife International, 2018), especially since wintering records in Pakistan are occasional (BirdLife International, 2001), and these, and those from north-east India, do not capture the whereabouts of most of the eastern flyway wintering population (Khan et al., 2017). Since hunting remains a key threat along migration routes of Sociable lapwing (Sheldon et al., 2013), the presence of the military in the DVNP could act as a deterrent to any such activities at this site. These records promote the importance of further surveys, guided by remote sensing, to locate other potential wintering grounds in the AJ and $\mathrm{K}$ region, especially those indicative of their wintering habitat requirements; dry plains and short grasslands (del Hoyo et al., 1996; BirdLife International, 2001).

DVNP is the only site within AJ and $\mathrm{K}$ that Red junglefowl is known to exist (Subhani et al., 2010). Our site and overall densities $\left(0.5 \pm 0.12 \mathrm{birds} / \mathrm{km}^{2}\right)$ are substantially less than those calculated in 2012 (Deva $=6.3$, Barmala $=8.8$ and Vatala $=15.6$ birds $/ \mathrm{km}^{2}$; Park density $=7.9$ birds $/ \mathrm{km}^{2}$ ), even when factoring in one encounter equating to four birds, based on its polygamous behaviour (Subhani et al., 2010). It is not known whether this is a genuine decline in the species population or a reflection of different survey methods used (call counts versus line transects). This requires further investigation due to the geographic importance of this site within the species' range (Subhani et al., 2010).

This also serves as a validatory point for standardising survey methods to maximise monitoring value. While species-specific methods may be appropriate (Bibby et al., 2000), comparable community assessment and monitoring will benefit from a single, standardised approach. In this case, line transects are already established within the Park. Applying distance sampling to transects (Buckland et al., 2001), rather than a fixed-width approach, will model detectability differences across multiple species, enabling robust single species abundance estimation, while providing an appropriate framework for community composition analysis; we recommend using the analysis framework used in this study (see Clarke, 1993; Clarke and Warwick, 2001). Even in the absence of more advanced abundance and composition analyses, using simple measures of relative abundance (e.g. Awan et al., 2012) as population indices (Gregory et al., 2004), provides a cost-effective management tool (Gamfeldt et al., 2008) for monitoring relative changes in species abundance within the National Park (Robertson and Liley, 1998). These can be utilised alongside conservation actions relating to and informing effective protected area management (Underhill and Gibbons, 2002; Gamfeldt et al., 2008) and community engagement and outreach (Berger et al., 2014).

Habitat degradation in the National Park is caused by 
a combination of grazing, wood cutting, grass collection, clearance by fire for agricultural land, and quarrying (Anwar et al., 2015). There is evidence to suggest that anthropogenic use of natural resources is increasing within the core zone, specifically for fuel wood and livestock grazing (Akrim et al., 2015), with livestock, e.g. goats, cows, moved into DVNP for grazing in the winter months (Anwar et al., 2015). Local hunters and egg collectors also utilise the DVNP, e.g. for Red junglefowl (Akrim et al., 2015) and Indian peafowl (Anwar et al., 2015). Hunting pressure may also impact Sociable lapwing, as elsewhere in its wintering range in Pakistan (Khan et al., 2017), although military presence may help reduce any potential threat. Quarrying is also reported and known to impact Indian peafowl in the landscape (Anwar et al., 2016). The Vatala range is easily accessible to local communities, while the deterrent of military presence in the Deva range, and proximity to the line of control, affords a degree of biodiversity and landscape protection (Anwar et al., 2016). This may also serve to help minimise impacts in the core zone and maintain an important ecotone of sub-montane forest and agricultural plains. Increasing awareness-raising and public engagement in local communities (Berger et al., 2014) and enforcing wildlife law are required to reduce anthropogenic impacts and support long-term conservation targets within the Park. Particular focus on the accessible Vatala range and localities where Sociable lapwing are recorded would potentially provide greatest conservation benefits.

\section{ACKNOWLEDGEMENTS}

We thank the staff of the AJ and K Forest Department for helping organise the fieldwork and collection of field data. We are grateful to Mr Abdul Razaq (Lecturer in Zoology) for his support and guidance in data collection, and MPhil Scholars and staff in the Department of Zoology, University of Gujrat for their support in collecting field data.

\section{Statement of conflict of interest}

The authors have declared no conflict of interest.

\section{REFERENCES}

Akrim, F., Awan, M.S., T. Mahmood, T., Anjum, M.Z., Qasim, S., Khalid, J. and Andleeb, S., 2015. Threats to Red Junglefowl (Gallus gallus murghi) in Deva Vatala National Park, District Bhimber, Azad Jammu and Kashmir, Pakistan. Annu. Res. Rev. Biol., 6: 59-65. https://doi.org/10.9734/ ARRB/2015/9596
Anwar, M., Ali, S., Rais, M. and Mahmood, T., 2016. Breeding ecology of red jungle fowl (Gallus gallus) in Deva Vatala National Park, Azad Jammu and Kashmir, Pakistan. J. Appl. Agric. Biotechnol., 1: 59-65. https://doi.org/10.9734/ARRB/2015/9596

Anwar, M., Mahmood, A., Rais, M., Hussain, I., Ashraf, N. and Khalil, S., 2015. Population density and habitat preference of Indian peafowl (Pavo cristatus) in Deva Vatala National Park, Azad Jammu and Kashmir, Pakistan. Pakistan J. Zool., 47: 1381-1386.

Awan, M.N., Ali, H. and Lee, D.C., 2012. An annotated checklist of birds and conservation issues in Salkhala Game Reserve, an isolated Important Bird Area in Azad Kashmir, Pakistan. Forktail, 28: 3843.

Azam, M.M., Hussain, R., Ali, M., Ahmed, W. and Abbas, N., 2007. Some observations on the biodiversity of Deva and Vatala Game Reserves, District Bhimber, AJK. Unpublished report. pp. 21.

Berger, J., Cain, S.L., Cheng, E., Dratch, P., Ellison, K., Francis, J., Frost, H.C., Gende, S., Groves, C., Karesh, W.A. and Leslie, E., 2014. Optimism and challenge for science-based conservation of migratory species in and out of US National Parks. Conserv. Biol., 28: 4-12.

Bibby, C., Burgess, N., Hill, D. and Mustoe, S., 2000. Bird Census Techniques, $2^{\text {nd }}$ edition Academic Press, San Diego, CA.

BirdLife International, 2001. Threatened Birds of Asia: The BirdLife International Red Data Book. BirdLife International, Cambridge, UK.

BirdLife International, 2018. Vanellus gregarius. The IUCN Red List of Threatened Species 2018: e.T22694053A130586388. Downloaded on 19 March 2020.

Buckland, S.T., Anderson, D.R., Burnham, K.P., Laake, J.L., Borchers, D.L. and Thomas, L., 2001. Introduction to Distance Sampling: Estimating Abundance of Biological Populations. Oxford University Press, N.Y.

Clarke, K. and Gorley, R., 2006. PRIMER v6: user manual/tutorial, Primer E: Plymouth. Plymouth Marine Laboratory, Plymouth, UK.

Clarke, K.R. and Warwick, R.M., 2001. Change in Marine Communities: An Approach to Statistical Analysis and Interpretation. Second edition. PRIMER-E, Plymouth, UK.

Clarke, K.R., 1993. Non-parametric multivariate analyses of changes in community structure. Aust. J. Ecol., 18: 117-143. https://doi. org/10.1111/j.1442-9993.1993.tb00438.x 
del Hoyo, J., Elliott, J. and Sargatal, 1996. Handbook of the Birds of the World, Vol. 3: Hoatzin to Auks. Lynx Edicions, Barcelona, Spain.

Gamfeldt, L., Hillebrand and Jonsson, 2008. Multiple functions increase the importance of biodiversity for overall ecosystem functioning. Ecology, 89: 1223-1231. https://doi.org/10.1890/06-2091.1

GOAJ\&K, 1985. Wildlife in Azad Jammu and Kashmir. Wildlife Wing, Forest Department, Azad Government of the State of Jammu and Kashmir, Muzaffarabad. pp. 53.

Gregory, R.D., Gibbons, D.W. and Donald, P.F., 2004. Bird census and survey techniques. In W.J. Sutherland, I. Newton and R.E. Green, eds. Bird Ecology and Conservation: A Handbook of Techniques. pp. 17-55. Oxford University Press, Oxford, UK. https://doi.org/10.1093/ acprof:oso/9780198520863.003.0002

Grimmett, R., Roberts, T.J., Inskipp, T. and Byers, C., 2008. Birds of Pakistan. A and C Black.

Khan, M.S., Sheldon, R. and Khan, A., 2017. Assessing the importance of Pakistan for wintering populations of the Critically Endangered Sociable Lapwing. Unpublished project report. Saiban Development Foundation, Pakistan.

Ladin, Z.S., Higgins, C.D., Schmit, J.P., Sanders, G., Johnson, M.J., Weed, A.S., Marshall, M.R., Campbell, J.P., Comiskey, J.A. and Shriver, W.G., 2016. Using regional bird community dynamics to evaluate ecological integrity within national parks. Ecosphere, 7: e01464. 10.1002/ecs2.1464. https:// doi.org/10.1002/ecs2.1464

MacKinnon, J. and Phillipps, K., 2005. A Field Guide to the Birds of China. Reprint. Oxford University Press, Oxford, UK.

Rasmussen, P.C. and Anderton, J.C., 2005. Birds of South Asia: the Ripley Guide. Washington, DC.

Robertson, P.A. and Liley, D., 1998. Assessment of sites: measurement of species richness and diversity. In C.J. Bibby, M.J. Jones and S.J. Marsden, eds. Expedition Field Techniques: Bird Surveys. pp. 76-98. Expedition Advisory Centre, Royal Geographical Society, London.

Sheldon, R.D., Kamp, J., Koshkin, M.A., Urazaliev, R.S., Iskakov, T.K., Field, R.H., Salemgareev, A.R., Khrokov, V.V., Zhuly, V.A. and Sklyarenko, S.L., 2013. Breeding ecology of the globally threatened Sociable Lapwing Vanellus gregarius and the demographic drivers of recent declines. $J$. Ornithol., 154: 501-516. https://doi.org/10.1007/ s10336-012-0921-4

Snow, D. and Perrins, C., 1998. The Birds of the Western Palearctic. Concise edition, Vols. I and II. Oxford University Press, Oxford, UK.

Subhani, A., Awan, M.S. and Anwar, M., 2010. Population status and distribution pattern of Red Jungle Fowl (Gallus gallus murghi) in Deva Vatala National Park, Azad Jammu and Kashmir, Pakistan: A pioneer study. Pakistan J. Zool., 42: 701-706.

Underhill, L. and Gibbons, D., 2002. Mapping and monitoring bird populations: their conservation uses. In K. Norris and D. J. Pain, eds. Conserving Bird Biodiversity: General Principles and their Application. pp. 34-60. Cambridge University Press, Cambridge, UK. https://doi.org/10.1017/ CBO9780511606304.004

Wathen, S., Thorne, J.H., Holguin, A. and Schwartz, M.W., 2014. Estimating the spatial and temporal distribution of species richness within Sequoia and Kings Canyon National Parks. PLoS One, 9: e112465. https://doi.org/10.1371/journal. pone. 0112465

Wiersma, P. and Kirwan, G.M. 2016. Red-wattled Lapwing (Vanellus indicus). In: Handbook of the birds of the world alive (eds. J. del Hoyo, A. Elliott, J. Sargatal, D.A. Christie and E. de Juana) Lynx Edicions, Barcelona. 